\title{
ON THE MAXIMISATION OF THE ADJUSTMENT COEFFICIENT UNDER PROPORTIONAL REINSURANCE
}

\author{
BY
}

\author{
Morten Hald And Hanspeter Schmidli
}

\begin{abstract}
In this note we consider how to maximise the adjustment coefficient in the case of proportional reinsurance. This complements some work of Waters (1983), where it was shown that there is a unique retention level maximising the adjustment coefficient. The advantage of our method is that only one implicit equation has to be solved.
\end{abstract}

\section{KEYWORDS}

Adjustment coefficient; proportional reinsurance; Cramér-Lundberg risk model; Sparre-Andersen risk model; Markov modulated risk model.

\section{INTRODUCTION}

Application of control theory to non-life insurance problems has obtained some attention recently. One of the problems considered was the minimisation of the ruin probability, see Hipp \& Plum (2000), Hipp \& Taksar (2000), Schmidli (2001) and Schmidli (2002a). In these papers it is shown that the value function fulfils the so-called Hamilton-Jacobi-Bellman equation. The optimal strategy can be obtained from the solution to the Hamilton-Jacobi-Bellman equation. In Hipp \& Schmidli (2002), see also Schmidli (2002b), it is shown that the optimal strategies converge as the initial capital tends to infinity. The asymptotic value can be found by maximising the adjustment coefficient.

Already Waters (1983) investigated the maximisation of the adjustment coefficient. He considered a model with an embedded regenerative structure. The reinsurance treaty was parametrised by a parameter $b$, and the corresponding adjustment coefficient was denoted by $R(b)$. Under quite mild conditions he proved that $b \mapsto R(b)$ is a unimodal function. Moreover, it was shown that there is a unique $b_{0}$ for which $R\left(b_{0}\right)=\sup _{b} R(b)$.

Because a model only is an approximation to reality and the calculation of the optimal strategy is quite complicated it seems reasonable to use a fixed strategy instead of a dynamic one. It seems to be simpler just to calculate the asymptotic value of the optimal strategy than to solve the Hamilton-Jacobi-Bellman 
equation and then to calculate the optimal strategy. Moreover, the adjustment coefficient is a stable measure for the risk.

The question how to calculate $b_{0}$ and $R\left(b_{0}\right)$ was not considered in Waters (1983). The problem is that the adjustment coefficient is given implicitly. A naive way would be to calculate $R(b)$ for different values of $b$. Because the function $R(b)$ is unimodal the area where $b_{0}$ lies can be localised better and better. Alternatively, Newton's method could be used to solve $R^{\prime}(b)=0$, but the second derivative is quite complicated. The problem is that $R(b)$ has to be found numerically, for example by Newton's method. This means that the naive method is time consuming.

In this paper we consider proportional reinsurance. We show how to calculate the maximal adjustment coefficient and the maximising parameter $b_{0}$ in an efficient way. We also prove that our method has a unique solution, and that this solution really coincides with $b_{0}$ and $R\left(b_{0}\right)$. The results had also been obtained in the first author's Master thesis Hald (2000). This note is organised as follows. In Section 2 we consider the classical Cramér-Lundberg model. We show that $b_{0}$ and $R\left(b_{0}\right)$ can be obtained by solving a simple equation. In the Sparre-Andersen model (Section 3) the equation to solve is a little bit more complicated, but there is still one implicit equation to be solved. Finally, in Section 4 we briefly discuss by way of two examples how more complicated models can be treated by the same ideas.

\section{THe Cramér-Lundberg MOdel}

Consider a model of the form

$$
X_{t}=u+c t-\sum_{i=1}^{N_{t}} Y_{i}
$$

where $u$ is the initial capital, $c$ is the (linear) premium rate, $\left\{N_{t}\right\}$ is a Poisson process with rate $\lambda$, say, and $\left\{Y_{i}\right\}$ are the claim sizes, assumed to be iid. We assume that $\left\{N_{t}\right\}$ and $\left\{Y_{i}\right\}$ are independent. We denote by $G(y)$ the distribution function of $Y_{i}$, by $\mu=\mathbb{E}\left[Y_{i}\right]$ its mean value and by $M_{Y}(r)=\mathbb{E}\left[\mathrm{e}^{r Y_{i}}\right]$ its moment generating function. The object of interest in ruin theory is the ruin probability $\psi(u)=\mathbb{P}\left[\inf _{t} X_{t}<0\right]$. In order that $\psi(u) \neq 1$ we have to assume the net profit condition $c>\lambda \mu$. For an introduction to the model see for instance Rolski et al. (1999).

The function $\theta(r)=\lambda\left(M_{Y}(r)-1\right)-c r$ is strictly convex. Because $\theta(0)=0$ and $\theta^{\prime}(0)=\lambda \mu-c<0$ there may be a value $R \neq 0$ such that $\theta(R)=0$. The solution $R$ is unique and strictly positive, and called the adjustment coefficient. For instance, $R$ exists if $\lim _{r \uparrow_{r_{\infty}}} M_{Y}(r)=\infty$, where $r_{\infty}=\sup \left\{r \geq 0: M_{Y}(r)<\infty\right\}$.

The fundamental results on the ruin probability in the small claim case are Lundberg's inequality

$$
\psi(u)<\mathrm{e}^{-R u}
$$


and the Cramér-Lundberg approximation

$$
\lim _{u \rightarrow \infty} \psi(u) \mathrm{e}^{R u}=\frac{c-\lambda \mu}{\lambda M_{Y}^{\prime}(R)-c} .
$$

The adjustment coefficient is therefore a measure of risk. For large values of $u$ it is possible to compare ruin probabilities by comparing the adjustment coefficients. For an actuary, it is therefore possible to base decisions upon comparison of adjustment coefficients.

Suppose the insurer has the possibility to choose proportional reinsurance with retention level $b \in[0,1]$. The premium rate for the reinsurance is $(1+\eta)$ $(1-b) \lambda \mu$. In order to simplify the notation we denote by $\kappa=c /(\lambda \mu)-1$ the safety loading of the insurer. Then the surplus process of the insurer becomes

$$
X_{t}^{b}=u+(b(1+\eta)-(\eta-k)) \lambda \mu t-\sum_{i=1}^{N_{t}} b Y_{i}
$$

with corresponding ruin probability $\psi^{b}(u)=\mathbb{P}\left[\inf _{t} X_{t}^{b}<0\right]$. If $\kappa \geq \eta$ the insurer can choose $b=0$ in order to obtain $\psi^{0}(u)=0$. In this case our problem becomes trivial. We therefore only consider the case $\eta>\kappa$, i.e. reinsurance is more expensive than first insurance.

In order that the net profit condition is fulfilled we need

$$
b>\underline{b}=1-\kappa / \eta \text {. }
$$

The adjustment coefficient $R(b)$ under reinsurance satisfies the equation

$$
\lambda\left(M_{Y}(b r)-1\right)-(b(1+\eta)-(\eta-\kappa)) \lambda \mu r=0 .
$$

Our goal is to maximise $R(b)$. We know from Waters (1983) that there is a unique $b_{0} \in[0,1]$ where the maximum is attained. For the moment we allow $b \in \mathbb{R}_{+}$. If the maximiser $b_{0} \notin[0,1]$ we know from the uni-modality that the optimal $b$ is 1 , i.e. no reinsurance is chosen.

The next result gives a simple way to calculate $b_{0}$ and $R\left(b_{0}\right)$.

Theorem 1. Assume that $r_{\infty}=\sup \left\{r: M_{Y}(r)<\infty\right\}>0$. Suppose there is a (unique) solution $\rho$ to

$$
M_{Y}^{\prime}(r)=(1+\eta) \mu .
$$

Then $\rho>0$. If no solution to (5) exists we let $\rho=r_{\infty}=\sup \left\{r: M_{Y}(r)<\infty\right\}$. For

$$
b_{0}^{\prime}=\frac{(\eta-\kappa) \mu \rho}{(1+\eta) \mu \rho+1-M_{Y}(\rho)}
$$

we have $b_{0}=b_{0}^{\prime} \wedge 1:=\min \left\{b_{0}^{\prime}, 1\right\}$. If $b_{0}=b_{0}^{\prime}$ then $R\left(b_{0}\right)=\rho / b_{0}$. 
Remark. Note that the value $\rho$ does not depend on $c$ but on the reinsurer's safety loading only.

Proof. Let $b_{0}^{\prime \prime}$ denote the argument where $R(b)$ attains its maximum in $\mathbb{R}_{+}$. Define $r(b)=b R(b)$. Then

$$
M_{Y}(r(b))-1+(\eta-\kappa) \mu \frac{r(b)}{b}-(1+\eta) \mu r(b)=0 .
$$

Because $R(b)$ is unimodal and continuous $r(b)$ is strictly increasing in $\left[\underline{b}, b_{0}^{\prime \prime}\right]$. Thus there exists $b_{1} \geq b_{0}^{\prime \prime}$ such that $b \mapsto r(b)$ is invertible on $\left[\underline{b}, b_{1}\right]$. We now interchange the rôles of $r$ and $b$. Then

$$
M_{Y}(r)-1+(\eta-\kappa) \mu \frac{r}{b(r)}-(1+\eta) \mu r=0 .
$$

$b(r)$ can be expressed as

$$
b(r)=\frac{(\eta-\kappa) \mu r}{(1+\eta) \mu r+1-M_{Y}(r)} .
$$

The adjustment coefficient as a function of $r$ becomes

$$
R(b(r))=\frac{r}{b(r)}=\frac{(1+\eta) \mu r+1-M_{Y}(r)}{(\eta-\kappa) \mu} .
$$

The function $r \mapsto R(b(r))$ is strictly concave, and has its unique maximum in $r=\rho$. Because $M_{Y}^{\prime}(0)=\mu<(1+\eta) \mu$ and $M_{Y}(r)$ is strictly convex we get that $\rho>0$. Because $\rho$ is unique it must coincide with $b_{0}^{\prime \prime} R\left(b_{0}^{\prime \prime}\right)$. Thus $b_{0}^{\prime}=b_{0}^{\prime \prime}$. Note that $r(b)>r_{\infty}$ is not possible. If $b_{0}^{\prime}>1$ then the maximum is attained at $b_{0}=1$.

Example 1. Suppose the claim sizes are gamma distributed with parameters $\gamma$ and $\beta$, i.e.

$$
G^{\prime}(y)=\frac{\beta^{\gamma}}{\Gamma(\gamma)} y^{\gamma-1} \mathrm{e}^{-\beta y} \mathbb{1}_{y>0},
$$

where $\mathbb{1}$ denotes the indicator function. The net profit condition yields $\beta c>\lambda \gamma$. Here $r_{\infty}=\beta$ and $\rho$ is obtained as the solution to

$$
\beta^{\gamma+1}=(1+\eta)(\beta-\rho)^{\gamma+1},
$$

i.e. $\rho=\beta\left(1-(1+\eta)^{-1 /(\gamma+1)}\right)$. From (6) we find

$$
b_{0}^{\prime}=\frac{\gamma(\eta-\kappa)\left(1-(1+\eta)^{-1 /(\gamma+1)}\right)}{\gamma \eta+(\gamma+1)\left(1-(1+\eta)^{\gamma /(\gamma+1)}\right)}
$$


yielding

$$
R\left(b_{0}^{\prime}\right)=\frac{\beta\left(\gamma \eta+(\gamma+1)\left(1-(1+\eta)^{\gamma /(\gamma+1)}\right)\right)}{\gamma(\eta-\kappa)}
$$

In this example there are, except for certain values of $\gamma$, no closed form expressions for $R(b)$. We see that even in this case it is possible to find closed form expressions for $b_{0}$ and $R\left(b_{0}\right)$ if $b_{0}^{\prime} \leq 1$.

\section{The SPARRE-ANDERSEN MODEL}

A generalisation of the Cramér-Lundberg model is the Sparre-Andersen model where $\left\{N_{t}\right\}$ is a renewal process. The interarrival times $\left\{T_{i}-T_{i-1}\right\}\left(T_{0}=0\right)$ are iid with distribution function $F(t)$. The mean value is denoted by $\lambda^{-1}=\mathbb{E}\left[T_{1}\right]$. The net profit condition is $\kappa>0$, where $\kappa$ is defined as in Section 2 . The adjustment coefficient $R$ is the (strictly positive) solution to

$$
M_{Y}(r) M_{T}(-c r)=1,
$$

where $M_{T}(r)=\mathbb{E}\left[e^{r T_{1}}\right]$ is the moment generating function of the interarrival times. Lundberg's inequality (1) remains valid and the Cramér-Lundberg approximation becomes $\lim _{u \rightarrow \infty} \psi(u) e^{R u}=C$ for some constant $C$, that in general is not known. Also here, the adjustment coefficient $R$ is a measure of risk. An introduction to the Sparre-Andersen risk model can be found for instance in Rolski et al. (1999).

We proceed in the same way as with the Cramér-Lundberg model. Under reinsurance the model is given by (3). The net profit condition yields $b>\underline{b}=$ $1-\kappa / \eta$. The adjustment coefficient $R(b)$ is the solution to

$$
M_{Y}(r b) M_{T}((\eta-\kappa-(1+\eta) b) \lambda \mu r)=1 .
$$

Theorem 2. Assume $r_{\infty}>0$. Suppose there is a unique solution $\rho$ in $\left(0, r_{\infty}\right)$ to

$$
M_{Y}^{\prime}(r)=(1+\eta) \lambda \mu M_{Y}(r)^{2} M_{T}^{\prime}\left(M_{T}^{-1}\left(1 / M_{Y}(r)\right)\right) .
$$

Then $\rho>0$. If no solution exists let $\rho=r_{\infty}$. Then with

$$
b_{0}^{\prime}=\frac{(\eta-\kappa) \rho \lambda \mu}{M_{T}^{-1}\left(1 / M_{Y}(\rho)\right)+(1+\eta) \rho \lambda \mu}
$$

we obtain $b_{0}=b_{0}^{\prime} \wedge 1$. If $b_{0}=b_{0}^{\prime}$ then $R\left(b_{0}\right)=\rho / b_{0}$. If the solution to (7) is not unique then $\rho=b_{0} R\left(b_{0}\right)$ solves (7).

Proof. Let again $r(b)=b R(b)$ and note that because of the uni-modality of $R(b)$ we have that $r(b)$ is invertible on an interval containing $\left[\underline{b}, b_{0}^{\prime \prime}\right]$, where $b_{0}^{\prime \prime}$ is the argument maximising $R(b)$ on $\mathbb{R}_{+}$. Then we need to solve 


$$
M_{Y}(r) M_{T}\left(\left((\eta-\kappa) \frac{r}{b(r)}-(1+\eta) r\right) \lambda \mu\right)=1,
$$

where we again interchange the rôles of $r$ and $b$. Then we can express $b(r)$ as

$$
b(r)=\frac{(\eta-\kappa) r \lambda \mu}{M_{T}^{-1}\left(1 / M_{Y}(r)\right)+(1+\eta) r \lambda \mu} .
$$

The adjustment coefficient as a function of $r$ is

$$
R(b(r))=\frac{r}{b(r)}=\frac{M_{T}^{-1}\left(1 / M_{Y}(r)\right)+(1+\eta) r \lambda \mu}{(\eta-\kappa) \lambda \mu} .
$$

By our assumption there is a unique maximum at $r=\rho$. The rest of the proof is analogous to the proof of Theorem 1.

Remark. If $1 / M_{Y}(r)$ is a concave function then $M_{T}^{-1}\left(1 / M_{Y}(r)\right)$ is strictly concave and a unique solution to (7) is granted. For instance, for gamma distributed claim sizes this is the case for $\gamma \leq 1$, in particular for exponentially distributed claim sizes.

From (7) one can see that the solutions will not be as explicit as in the case of the Cramér-Lundberg model.

Example 2. Suppose that the claim sizes are gamma distributed with parameters $\gamma$ and $\beta$ and the inter-arrival times are gamma distributed with parameters $g$ and $\alpha$. Then $\rho$ is the solution to

$$
\beta^{\gamma-1}\left(\alpha-((\beta-r) / \beta)^{\gamma}\right)^{g+1}=\alpha^{g+1}(1+\eta)(\beta-r)^{\gamma-1} .
$$

If $\gamma$ and $g$ are integer-valued one has to solve a polynomial equation from which $\rho, b_{0}$ and $R\left(b_{0}\right)$ can be obtained. If $\rho<\beta$ is not unique one has to try all solutions and then later to decide which solution is the correct one. For exponentially distributed claim sizes $(\gamma=1)$

$$
\rho=\beta\left(1-(g(1+\eta))^{-1 /(\gamma+1 / g)}\right)
$$

and

$$
b_{0}^{\prime}=\frac{\gamma(\eta-\kappa)\left(1-(g(1+\eta))^{-1 /(\gamma+1 / g)}\right)}{g\left(1-(g(1+\eta))^{-\gamma /(g+1)}+\gamma(1+\eta)\left(1-(g(1+\eta))^{-1 /(\gamma+1 / g)}\right)\right.} .
$$

If $b_{0}^{\prime} \leq 1$ then 


$$
R\left(b_{0}\right)=\frac{\beta g\left(1-(g(1+\eta))^{-\gamma /(\gamma g+1)}+\gamma(1+\eta)\left(1-(g(1+\eta))^{-1 /(\gamma+1 / g)}\right)\right.}{\gamma(\eta-\kappa)} .
$$

Also in this example $b_{0}$ and $R\left(b_{0}\right)$ are explicit if $b_{0}^{\prime} \leq 1$.

\section{EXTENSIONS}

The reason why it was so simple to find the optimal $b$ was that we were able to isolate $b$ as a function of $r$ from the equation determining $R(b)$. In our case $b$ appeared once in the formula only. Of course, our method also works if several solutions $b_{k}$ exist, and if it is possible to determine the correct $b$ (for example if $b$ appears in a quadratic equation). We just mention two cases where our method works.

\subsection{Non-linear premia in the Cramér-Lundberg model}

Suppose the insurer uses the variance premium principle, i.e. the premium becomes $(1-\mathrm{b}) \lambda \mu+v(1-\mathrm{b})^{2} \lambda \mu_{2}$ where $\mu_{2}=\mathbb{E}\left[Y_{i}^{2}\right]$. In order that the problem becomes non-trivial we have to assume that $c<\lambda\left(\mu+v \mu_{2}\right)$. The adjustment coefficient $R(b)$ is then determined through the equation

$$
\lambda\left(M_{Y}(r b)-1\right)+\left((1-b) \lambda \mu+v(1-b)^{2} \lambda \mu_{2}-c\right) r=0 .
$$

Again defining $r(b)=b R(b)$ we can write

$$
\lambda\left(M_{Y}(r)-1\right)+\left(\left(\frac{r}{b(r)}-r\right) \lambda \mu+v\left(\frac{r}{b(r)}-2 r+r b(r)\right) \lambda \mu_{2}-c \frac{r}{b(r)}\right)=0 .
$$

This gives

$$
b(r)=\frac{\mu+2 v \mu_{2}-\frac{M_{Y}(r)-1}{r} \pm \sqrt{\left(\mu+2 v \mu_{2}-\frac{M_{Y}(r)-1}{r}\right)^{2}-4 v \mu_{2}\left(\mu+v \mu_{2}-\frac{c}{\lambda}\right)}}{2 v \mu_{2}} .
$$

For $r>0$ both solutions are positive. As a side condition we need that the solutions are real and that $\left(M_{Y}(r)-1\right)-\left(\mu+2 v \mu_{2}\right) r<0$. The net profit condition yields that $b>\underline{b}=1-\sqrt{(c-\lambda \mu) /\left(\lambda \vee \mu_{2}\right)}$. For $b=\underline{b}$ we get $R(b)=0=r(b)$. Letting $r \downarrow 0$ we find

$$
b(0)=\frac{2 v \mu_{2} \pm \sqrt{4 v \mu_{2}\left(\frac{c}{\lambda}-\mu\right)}}{2 v \mu_{2}}
$$


Thus the solution with the minus sign applies. By continuity

$$
b(r)=\frac{\mu+2 v \mu_{2}-\frac{M_{f}(r)-1}{r}-\sqrt{\left(\mu+2 v \mu_{2}-\frac{M_{Y}(r)-1}{r}\right)^{2}-4 v \mu_{2}\left(\mu+v \mu_{2}-\frac{c}{\lambda}\right)}}{2 v \mu_{2}} .
$$

The function $r \mapsto R(b(r))$ can then be maximised. The expression becomes quite complicated, but the method goes through.

Remark. We also know the solution for $r=R(1)$, namely

$$
1=b(R(1))=\frac{\mu+2 v \mu_{2}-\frac{c}{\lambda} \pm \sqrt{\left(\frac{c}{\lambda}-\mu\right)^{2}}}{2 v \mu_{2}} .
$$

Thus the solution with the plus sign applies. The explanation must be that $b_{0}<1$. This was also observed by Waters (1983).

\subsection{The Markov modulated risk model}

Let $\left\{J_{t}\right\}$ be a Markov chain in continuous time on the state space $\{1,2, \ldots, g\}$ with intensity matrix $\boldsymbol{\eta}$. That is, as long as $J_{t}=i$ the process $\left\{J_{t}\right\}$ jumps after an exponentially distributed time with parameter $-\eta_{i i}$. The next state is $j$ with probability $-\eta_{i j} / \eta_{i i}$. The risk process $X$ is defined as follows. When $J_{t}=i$ the process behaves like a Cramér-Lundberg model with premium rate $c$, claim arrival intensity $L_{i}$ (i.e. claims arrive according to a Poisson process with rate $L_{i}$ ) and claim size distribution $G_{i}(y)$. For a formal definition of the model see for instance Rolski et al. (1999).

We denote by $\pi$ the stationary distribution of the Markov chain $\left\{J_{t}\right\}$, i.e. the solution to $\boldsymbol{\pi} \boldsymbol{\eta}=\mathbf{0}$ normed such that $\boldsymbol{\pi} \mathbf{1}=1$. The net profit condition becomes $c>\sum_{i=1}^{g} \pi_{i} L_{i} \mu_{i}$, where $\mu_{i}=\int_{0}^{\infty}\left(1-G_{i}(y)\right) d y$.

Let $\boldsymbol{S}(r)=\operatorname{diag}\left(L_{i}\left(M_{i}(r)-1\right)\right)$, where $M_{i}(r)=\int_{0}^{\infty} \mathrm{e}^{r y} \mathrm{~d} G_{i}(y)$, and $\boldsymbol{\Phi}(r)=\boldsymbol{\eta}+$ $\boldsymbol{S}(r)-c r \boldsymbol{I}$. Define by $\vartheta(r)$ the eigenvalue of $\boldsymbol{\Phi}(r)$ with the largest real part. By the Perron-Frobenius theorem this eigenvalue is uniquely defined. It turns out that $\vartheta(r)$ is a strictly convex function. The adjustment coefficient is defined as the non-zero solution $R$ to $\vartheta(r)=0$. If $R$ exists then Lundberg's inequality $\psi(u) \leq C_{+} \mathrm{e}^{-R u}$ holds. If $M_{i}^{\prime}(R)<\infty$ then also a Cramér-Lundberg approximation $\lim _{u \rightarrow \infty} \psi(u) \mathrm{e}^{R u}=C$ can be proved. For explicit expressions for $C_{+}$and $C$ see for instance Rolski et al. (1999). Thus again $R$ is a measure of risk.

Under proportional reinsurance the matrix $\boldsymbol{\Phi}$ becomes

$$
\boldsymbol{\Phi}(r ; b)=\boldsymbol{\eta}+\boldsymbol{S}(r b)+((\eta-\kappa)-b(1+\eta)) \sum_{i=1}^{g} \pi_{i} L_{i} \mu_{i} r \boldsymbol{I} .
$$

The adjustment coefficient $R(b)$ is then the solution to $\operatorname{det} \boldsymbol{\Phi}(r ; b)=0$. Again defining $r(b)=b R(b)$ and interchanging the rôles of $b$ and $r$ yields a polynomial 
for $b$ of degree $\mathcal{I}$. Therefore we obtain $\mathcal{I}$ solutions $b(r)$. Which solution to choose seems not to be obvious. But one can calculate $\rho$ for all the $\mathcal{I}$ solutions and decide afterwards which is the correct solution.

\section{ACKNOWLEDGEMENTS}

The authors thank two unknown referees for their useful comments on an earlier version of the paper.

\section{REFERENCES}

HALD, M. (2000) Optimal reinsurance forms given in terms of a maximised adjustment coefficient. Master Thesis, University of Aarhus.

HipP, C. and Plum, M. (2000) Optimal investment for insurers. Insurance Math. Econom. 27, 215-228.

HipP, C. and SchmidLi, H. (2002) Asymptotics of ruin probabilities for controlled risk processes in the small claims case. Working paper 186, Laboratory of Actuarial Mathematics, University of Copenhagen.

Hipp, C. and TAKSAR, M. (2000) Stochastic control for optimal new business. Insurance Math. Econom. 26, 185-192.

Rolski, T., Schmidli, H., Schmidt, V. and Teugels, J.L. (1999) Stochastic Processes for Insurance and Finance. Wiley, Chichester.

Schmidli, H. (2001) Optimal proportional reinsurance policies in a dynamic setting. Scand. Actuarial J., 55-68.

SCHMidLI, H. (2002a) On minimising the ruin probability by investment and reinsurance. Ann. Appl. Probab. 12, 890-907.

Schmidli, H. (2002b) Asymptotics of ruin probabilities for risk processes under optimal reinsurance policies: the small claim case. Working paper 180, Laboratory of Actuarial Mathematics, University of Copenhagen.

WATERS, H.R. (1983) Some mathematical aspects of reinsurance. Insurance Math. Econom. 2, 17 26.

\section{Morten HaLd \\ Codan \\ Codanhus \\ Gammel Kongevej 60 \\ DK-1790 Copenhagen V \\ Denmark}

HANSPETER SCHMIDLI

Laboratory of Actuarial Mathematics

University of Copenhagen

Universitetsparken 5

DK-2100 Copenhagen Ø

Denmark 